\title{
Procedimiento para la Reconstrucción de un Score Predictivo de Deterioro Grave en Pacientes Internados
}

\author{
Hernán José Navas, Elián Daniel Bourdin \\ Informática en Salud \\ Sanatorio Finochietto \\ Ciudad Autónoma de Buenos Aires, Argentina \\ \{hnavas, ebourdin\}@sanatoriofinochietto.com
}

\begin{abstract}
Resumen-Un sistema de información hospitalario es un sistema basado en computadoras, que facilita el manejo de la información asistencial y administrativa de un hospital, para mejorar la calidad y eficiencia del cuidado de la salud. Los sistemas de soporte a la toma de decisiones integran la información clínica y de los pacientes. Hasta el $\mathbf{8 0} \%$ de los pacientes en internación general tienen parámetros fisiológicos fuera de rangos normales $\mathbf{2 4}$ horas antes del traslado a la unidad de cuidados intensivos (UCI). De los sistemas de detección temprana de pacientes de riesgo, NEWS tiene mayor posibilidad de discriminar pacientes con riesgo de paro cardíaco, ingreso no previsto a UCI y muerte a partir de un score que pondera el desvío de 6 parámetros respecto a los rangos normales. Cualquier valor faltante dificulta la generación de alertas. Es frecuente encontrar registros incompletos. El procedimiento de reconstrucción permite obtener mayor cantidad de registros completos.
\end{abstract}

Palabras Clave-Procedimiento de reconstrucción de variables, Sistema de información hospitalario, Sistema de soporte a la toma de decisiones.

\section{INTRODUCCIÓN}

Las tecnologías de información y comunicación (TIC) en las últimas décadas, a través del uso de las computadoras e Internet, se han convertido en motor de cambio, y de desarrollo social y económico. El sector de la salud no ha quedado ajeno a esta situación. Sin embargo, los sistemas de salud a nivel global están sometidos a la tensión de aumentar cobertura y calidad, así como controlar los costos crecientes. En este escenario, las TIC están llamadas a contribuir a la solución del dilema y no podrán estar ausentes de las políticas de salud. Se han identificado ocho dimensiones de contribución de las TIC en el ámbito sanitario: acceso, eficacia, eficiencia, calidad, seguridad, generación de conocimiento, impacto en la economía e integración [1].

Un sistema de información hospitalario (HIS, Health Information System por sus siglas en inglés) puede definirse como un sistema basado en computadoras, diseñado para facilitar el manejo de toda la información de salud y administrativa de un hospital, con el objetivo de mejorar la calidad y eficiencia del cuidado de la salud. Con el tiempo, el HIS se convirtió en un gran repositorio de datos, capaz de brindar información, que correctamente empleada, puede servir para dar soporte a la toma de decisiones [2].

El desarrollo y crecimiento de los HIS necesitó de médicos especializados en el manejo de la información, dando lugar a una nueva disciplina denominada: informática en salud. Esta nueva especialidad abarca la comprensión, habilidades y herramientas que permitan el intercambio y la utilización de la información para proporcionar asistencia sanitaria y promoción de la salud. Es una disciplina académica definida y desarrollada en las últimas décadas por una comunidad mundial de científicos que participan en la promoción y enseñanza del conocimiento acerca de la aplicación de tecnologías de la información en la asistencia sanitaria, el lugar donde la salud, la información y las ciencias de la informática, la psicología, la epidemiología, y la ingeniería se cruzan [3].

Los sistemas de soporte a la toma de decisiones en el ámbito de la salud son sistemas de información de base informática que se utilizan para integrar la información clínica y la de los pacientes con el objetivo de respaldar la toma de decisiones en la atención de los pacientes. Pueden ser útiles para ayudar en el proceso de diagnóstico, la emisión de alertas y recordatorios, la crítica y planificación del tratamiento, la recuperación de información, y el reconocimiento y la interpretación de imágenes [4].

Es sabido que hasta el $80 \%$ de los pacientes que se encuentran hospitalizados en internación general tienen parámetros fisiológicos fuera de los rangos normales dentro de las 24 horas previas al traslado a la unidad de cuidados intensivos (UCI). La falta de pronta y adecuada respuesta al deterioro del paciente puede llevar a un aumento de su morbimortalidad y de los costos de atención [5].

Dentro de los múltiples sistemas de detección temprana de pacientes de riesgo, particularmente NEWS (uno de los sistemas de ponderación agregada) tiene mayor posibilidad de discriminar pacientes con riesgo de paro cardíaco, ingreso no previsto a UCI y muerte [6,7]. Se basa en la asignación de puntajes a determinadas variables fisiológicas en función del grado de desvío respecto del rango normal. La sumatoria del puntaje de cada variable da como resultado el valor del score que permite predecir el riesgo de deterioro dentro de las próximas 12 horas, dependiendo del resultado obtenido. Los parámetros fisiológicos que se consideran son: (i) frecuencia respiratoria, (ii) saturación de oxígeno, (iii) temperatura axilar, (iv) presión sanguínea sistólica, (v) pulso, (vi) nivel de consciencia [8].

El Sanatorio Finochietto es una institución privada de salud, inaugurada en noviembre de 2013, con 125 camas de internación general para adultos, 31 de unidad de cuidados intensivos para adultos y 16 puestos de cuidados intensivos neonatales. Es el primer centro asistencial bio-eco-inteligente de Argentina, certificado como miembro de la Red global de hospitales verdes y saludables. Posee un HIS comercial que 
permite el manejo de la comunicación por vía electrónica entre todo el equipo de salud (médicos, kinesiólogos, enfermeros, farmacéuticos, nutricionistas, etc.), y el personal administrativo. Posee interfaces con los efectores de estudios complementarios, como laboratorio, diagnóstico por imágenes, endoscopía, etc. y utiliza dispositivos móviles para la administración segura de medicamentos y para el registro de información al pie de la cama del paciente [9].

En este contexto, en el presente artículo se presenta la delimitación del problema en la sección II, la solución propuesta en la sección III, la validación de los resultados en la sección IV, con la descripción de los materiales y métodos en la sección IV.A, los resultados y su interpretación en la sección IV.B, finalizando con la conclusión en la sección V.

\section{PROBLEMA}

El cálculo del score para la predicción del deterioro depende de la existencia de los 6 parámetros, cualquier valor faltante genera dificultades en la generación de alertas como consecuencia de un error en dicho cálculo [10, 11].

La utilización de estos métodos de evaluación de pacientes para predecir riesgo no se encuentra ampliamente distribuida. En hospitales universitarios con médicos y enfermeros en formación es más probable su implementación ya que es necesaria una capacitación constante del personal, aunque también en algunos casos resulta dificultosa [12].

En un estudio previo, los autores desarrollaron una estrategia que permitió relacionar los valores para evitar faltantes, mediante la creación arbitraria de franjas horarias de dos horas cada una. En cada franja, un algoritmo localizaba todos los parámetros y los combinaba de todas las formas posibles, informando solamente el score más alto calculado [13]. Si bien se lograron recomponer mayor cantidad de scores, la cantidad impresionó baja, probablemente debido a la rigidez de los límites de cada franja horaria.

\section{SOLUCIÓN PROPUESTA}

Luego de analizar los resultados del estudio previo se observó que uno de los parámetros, la valoración de la consciencia, era el que menor cantidad de mediciones tenía por lo cual era el factor limitante para la reconstrucción.

A continuación, se describe el procedimiento a emplear en la sección III.A y se brinda un ejemplo con un caso en la sección III.B.

\section{A. Descripción del procedimiento}

En esta sección se presentan los pasos a seguir:

Paso 1: Realizar la búsqueda de los parámetros a analizar en la base de datos del HIS, con la fecha de medición y el paciente al que corresponde.

Paso 2: Almacenar cada parámetro en una tabla diferente, manteniendo la fecha y el paciente.

Paso 3: Tomando como referencia cada uno de los valores del parámetro de consciencia registrado, asociar entre sí los datos de cada una de las tablas, en función del identificador único del paciente y del horario de toma de mediciones, con un máximo de una hora de diferencia. El margen de una hora se establece para que los datos posean significancia clínica.

Paso 4: Eliminar las líneas exactamente iguales para mejorar la performance del procesamiento futuro de la información.

Paso 5: Calcular un valor de score para cada una de las combinaciones posibles de cada conjunto de 6 parámetros de un mismo paciente.
En la imagen que sigue (Fig. 1) puede observarse el problema y cómo debe realizarse la combinación para el cálculo de los scores. Dado un mismo paciente llamado " $\mathrm{A}$ " en ninguna de las 4 mediciones (cada fila representa una medición) se encuentran los 6 parámetros completos. Cada medición se agrupa en función del valor medido para la consciencia. Tomando como punto de partida este último parámetro se deben recorrer los restantes realizando todas las combinaciones necesarias para completar los parámetros faltantes para cada una de las mediciones. De esta manera la cantidad de scores por fila dependerá de la cantidad de combinaciones que se hayan realizado para completar los parámetros faltantes.

Paso 6: Agrupar los registros para cada paciente.

Paso 7: Ante la existencia de múltiples mediciones combinadas para un parámetro de consciencia en particular, mantener el score más alto, desestimando el resto de los registros.

Paso 8: Ordenar los registros en función del momento en el que fueron realizados. Esto facilitará el análisis de la progresión del deterioro del paciente.

\section{B. Prueba de concepto}

Para ilustrar el procedimiento se describe el caso del paciente 10793. Inicialmente, a través de la recolección en la base de datos, se obtuvieron 4.045 combinaciones de parámetros, en función de múltiples evaluaciones de consciencia, desde el inicio de la internación hasta el momento del traslado nocturno a la UCI.

Se desarrollaron 2 scripts, el primero para resolver el paso 5 , y el restante los pasos 6 al 8 .

En una primera instancia, se aplicaron los filtros de valores repetidos. Esto ayudó significativamente al procesamiento de la información, debido a la eliminación de líneas exactamente iguales. Quedaron 730 combinaciones diferentes de parámetros. Luego de este procedimiento, se ejecutó el primer script para el cálculo del score de cada una de las combinaciones reconstruidas.

Finalmente, se ejecutó el segundo script, para agrupar todas las mediciones asociadas a una evaluación de consciencia en particular y considerar el resultado más alto para cada una. Además, el script ordenó cronológicamente la información de salida para facilitar el análisis de la progresión del deterioro del paciente.

Como resultado final, para el paciente 10793 se obtuvieron 7 reconstrucciones de score, de los cuales 2 se encontraron dentro de las 12 horas previas al traslado a UCI (Tabla 1).

TABLA 1. EJEMPLO DE SALIDA PARA UN PACIENTE DETERMINADO

\begin{tabular}{ccc}
\hline Paciente & $\begin{array}{c}\text { Cantidad de horas previas al } \\
\text { traslado a UCl }\end{array}$ & Score \\
\hline 10793 & 141 & 6 \\
10793 & 96 & 17 \\
10793 & 91 & 12 \\
10793 & 48 & 7 \\
10793 & 43 & 6 \\
10793 & 11 & 5 \\
70793 & 2 & 11 \\
\hline
\end{tabular}

IV. VALIDACIÓN DE LA SOLUCIÓN

En esta sección se presentarán los materiales y métodos utilizados para validar la solución propuesta en la sección IV.A y se presentarán e interpretarán los resultados obtenidos en la sección IV.B. 


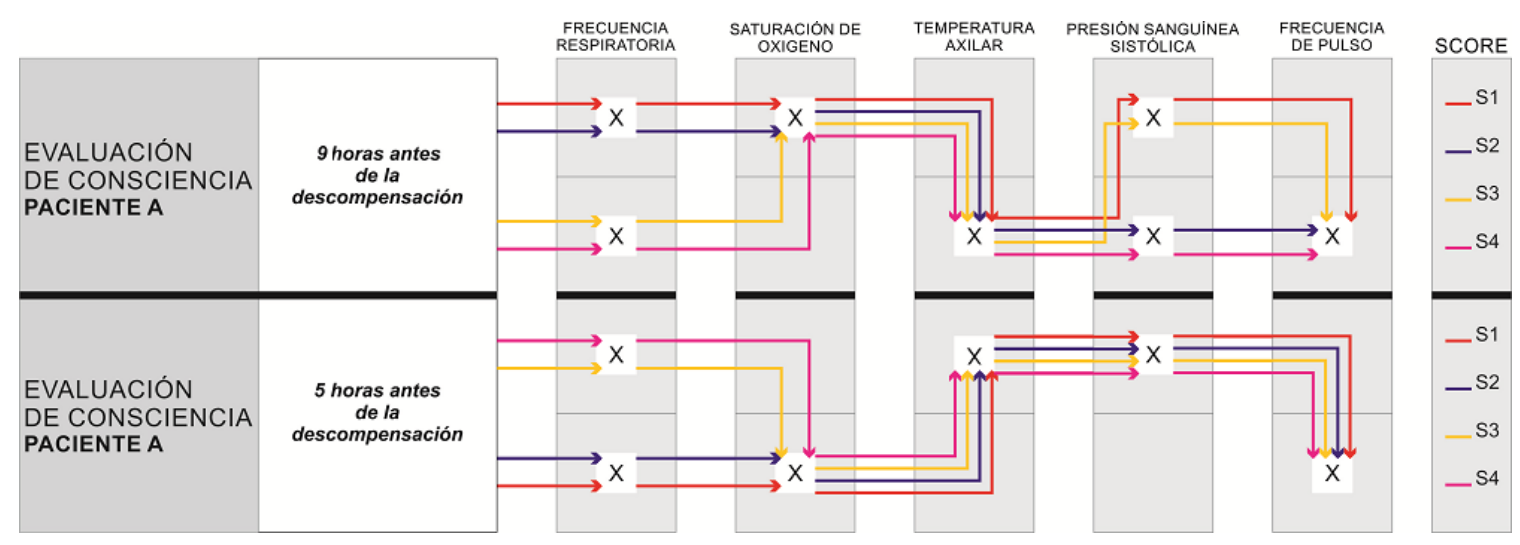

Fig. 1. Ejemplo de reconstrucción de Score

\section{A. Materiales y métodos}

Se utilizó exactamente el mismo conjunto de datos que para el estudio previo. Todos los pacientes que fueron trasladados desde internación general hacia la unidad de cuidados intensivos en el horario comprendido entre las 22 y las 5 de la mañana, desde el 14/11/2013 hasta el 09/05/2016. La selección del horario representa necesariamente pacientes que se descompensaron repentinamente durante su estadía en el área de internación general y requirieron un traslado de urgencia a la UCI para aumentar el nivel de complejidad de su atención. No existen movimientos programados de pacientes en dicha franja horaria.

El periodo de recolección de datos para cada paciente se realizó desde el inicio de la internación y hasta el momento del traslado a la unidad de cuidados intensivos en la franja horaria seleccionada.

Ambos scripts fueron programados en PERL, por ser un lenguaje de propósito general, originalmente desarrollado para la manipulación de texto, pero que actualmente se utiliza para un amplio rango de tareas, gracias a su facilidad de manejo, su versatilidad y potencia [14].

\section{B. Resultados e interpretación}

Los 283 pacientes analizados generaron 20.864 .012 de líneas candidatas para calcular el score a partir de todas las combinaciones posibles de parámetros, siguiendo las condiciones de pertenecer a un mismo paciente y encontrarse en el rango de una hora en más o en menos tomando como eje al valor de la consciencia.

El procesamiento de todas las combinaciones generadas por parte del segundo script entregó un total de 1.244 scores pertenecientes a 110 pacientes.

Finalmente, y para poder comparar con el método empleado en el estudio previo [13], se filtraron los scores de las últimas 12 horas, previas al traslado a la UCI. El resultado obtenido fue de 79 scores de 56 pacientes.

El proceso manual de recolección de los parámetros se realiza en 2 pantallas diferentes del sistema; precisamente el parámetro detectado como limitante es el que se encuentra separado del conjunto. Según los datos analizados, primero se registra el estado de la consciencia y luego los 5 parámetros restantes (los parámetros fisiológicos).

Para representar el proceso manual se realizó una búsqueda que asoció los parámetros físiológicos con el estado de la consciencia en un rango no mayor a 10 minutos. Siguiendo los mismos criterios que los empleados para las reconstrucciones se detectaron 4 mediciones de 4 pacientes diferentes (Tabla 2).
TABLA 2. RECUPERACIÓN DE DATOS POR MÉTODO

\begin{tabular}{cccc}
\hline Método & \multicolumn{2}{c}{ Pacientes } & Scores \\
\hline & Cantidad & $\begin{array}{c}\% \text { del } \\
\text { set de } \\
\text { datos }\end{array}$ & Cantidad \\
\hline Manual & 4 & $1 \%$ & 4 \\
$\begin{array}{c}\text { Franjas } \\
\text { horarias }\end{array}$ & 41 & $15 \%$ & 43 \\
$\begin{array}{c}\text { Parámetro } \\
\text { limitante }\end{array}$ & 56 & $20 \%$ & 79 \\
\hline
\end{tabular}

El sistema de soporte a la toma de decisiones necesita de la existencia de scores para generar las alarmas. Para el método manual habrían existido sólo 4 scores para analizar y generar las alertas correspondientes, en cambio la disponibilidad de scores aumentó sustancialmente con el método del parámetro limitante a 79. La mayor disponibilidad de scores que de pacientes significa que, dado un mismo paciente, existe más de una reconstrucción dentro de las 12 horas previas al evento. Desde el punto de vista asistencial esto significa una gran ventaja ya que permite analizar la tendencia hacia el deterioro en cada paciente con más de una reconstrucción.

Se detectaron algunos casos con scores muy elevados en momentos alejados del traslado a UCI que guardan relación con una estancia previa del paciente en dicha área. Allí cada paciente se encuentra conectado a un monitor multiparamétrico que envía constantemente los parámetros vitales al HIS. Esta situación fue la que provocó también la elevada cantidad de líneas encontradas inicialmente.

Finalmente se compararon los 4 pacientes que poseían los registros que representaban al proceso manual, con los obtenidos por el método del parámetro limitante. Se encontró una concordancia exacta en 3 de los 4 pacientes. El valor diferente fue de solamente 1 punto; se obtuvo un 9 con el método manual y un 10 con la reconstrucción mediante el parámetro limitante. Esto se encuentra dentro de lo esperado ya que el método propuesto mantiene solamente el mayor valor posible de la combinación encontrada (Tabla 3).

TABLA 3. RECUPERACIÓN DE DATOS POR MÉTODO

\begin{tabular}{ccc}
\hline Paciente & Modo manual & Reconstrucción \\
\hline 22010 & 10 & 10 \\
10793 & 9 & 10 \\
24794 & 5 & 5 \\
83004 & 3 & 3 \\
\hline
\end{tabular}




\section{CONCLUSIÓN}

Los sistemas de soporte a la toma de decisiones en el ámbito de la salud proporcionan una base objetiva $\mathrm{y}$ estandarizada para el cuidado de los pacientes. Particularmente los sistemas de detección temprana de deterioro permiten reducir el riesgo de muerte o complicaciones para el paciente, y los costos de atención asociados. El score de NEWS se basa en la evaluación de 6 parámetros para la predicción de dicho deterioro. La inexistencia de al menos un parámetro de los necesarios para realizar el cálculo dificulta la obtención del score generando una demora innecesaria en la atención, con consecuencias eventualmente fatales.

La cantidad de mediciones realizadas por completo en el mismo momento son escasas pero los resultados del método propuesto para el cálculo de esas mismas mediciones son prometedores ya que la coincidencia es del 75\%. Para el valor restante si bien se sobreestimó en el método propuesto, fue por decisión propia de los investigadores dada la sensibilidad de la información a tratar y el contexto de tratamiento de la misma. El procedimiento del tratamiento de la información descripto permite mejorar la disponibilidad de información en más de 10 veces con respecto a la cantidad de pacientes y casi 20 veces para la cantidad de scores de éstos, teniendo en cuenta las formas de registro actuales. Esta información permitirá disminuir los riesgos de eventos graves en los pacientes hospitalizados al ser intervenidos a tiempo, a pesar de no contar inicialmente con todos los valores completos.

Creemos que se podría obtener mayor cantidad de mediciones del parámetro limitante si se realizara un cambio en las pantallas donde se registra esta información ya que se encuentra en una pantalla diferente al resto de los 5 parámetros y en otro contexto. Según el método descripto no haría falta la medición de los 6 parámetros completos, pero sí podría cambiar la cantidad de información recuperada si se tuvieran más mediciones del valor de la consciencia.

Si bien los pacientes recuperados del HIS son pacientes descompensados, en cuyo caso la reconstrucción del score en las horas previas es de suma importancia, existe otro grupo de pacientes que se descompensaron durante el día y fueron trasladados a UCI que no fueron tenidos en cuenta.

Se prevé continuar este trabajo con la validación del procedimiento de reconstrucción propuesto contra el proceso manual de medición de los 6 parámetros para un mismo momento. Se realizará el cálculo según el método descripto en un determinado horario del día y sobre los mismos pacientes calculados, un enfermero entrenado, sin conocer los valores existentes, realizará las mediciones y registros de los 6 parámetros de cada paciente. Finalmente se calculará el score para ambos métodos y se analizarán las diferencias.

\section{FINANCIAMIENTO}

La presente investigación se realizó con financiamiento parcial del Departamento de Informática en Salud del Sanatorio Finochietto. Las opiniones de los autores no reflejan la posición oficial de la Institución.

\section{AGRADECIMIENTOS}

En primer lugar, queremos agradecerle al Dr. Kritzer por haber sido la fuente de inspiración sobre el tema. Y hacemos una especial mención al Dr. Ramón García Martínez por habernos apadrinado dedicando tiempo para corregirnos y guiarnos en el análisis y presentación de nuestra investigación.

\section{REFERENCIAS}

[1] González Bernaldo de Quirós F, Luna D., Baum A., Plazzotta F., Otero C. and Benitez S.: Incorporación de tecnologías de la información y de las comunicaciones en el Hospital Italiano de Buenos Aires. Naciones Unidas, Santiago de Chile (2012).

[2] Glaser, J. and Overhage, J.M.: The role of healthcare IT: becoming a learning organization. Healthc. Financ. Manag. J. Healthc. Financ. Manag. Assoc. 67, 56-62, 64 (2013).

[3] Shortliffe, E.H. and Blois $\dagger$, M.S.: The Computer Meets Medicine and Biology: Emergence of a Discipline. In: Shortliffe, E.H. and Cimino, J.J. (eds.) Biomedical Informatics: Computer Applications in Health Care and Biomedicine. pp. 345. Springer New York, New York, NY (2006).

[4] Beeler, P.E., Bates, D.W. and Hug, B.L.: Clinical decision support systems. Swiss Med. Wkly. 144, w14073 (2014).

[5] Tarassenko, L., Hann, A. and Young, D.: Integrated monitoring and analysis for early warning of patient deterioration. Br. J. Anaesth. 97, 64-68 (2006).

[6] Churpek, M.M., Yuen, T.C. and Edelson, D.P.: Risk stratification of hospitalized patients on the wards. Chest. 143, 1758-1765 (2013).

[7] Smith, G.B., Prytherch, D.R., Meredith, P., Schmidt, P.E and Featherstone, P.I.: The ability of the National Early Warning Score (NEWS) to discriminate patients at risk of early cardiac arrest, unanticipated intensive care unit admission, and death. Resuscitation. 84, 465-470 (2013).

[8] Royal College of Physicians of London: National Early Warning Score (NEWS): standardising the assessment of acute-illness severity in the NHS. Royal College of Physicians, London (2012).

[9] Navas, H., Graffi Moltrasio, L., Ares, F., Strumia, G., Dourado, E. and Alvarez, M.: Using mobile devices to improve the safety of medication administration processes. Stud. Health Technol. Inform. 216, 903 (2015).

[10] Clifton, D.A., et al.: "Errors" and omissions in paper-based early warning scores: the association with changes in vital signs--a database analysis. BMJ Open. 5, e007376 (2015).

[11] Gordon, C.F.and Beckett, D.J.: Significant deficiencies in the overnight use of a Standardised Early Warning Scoring system in a teaching hospital. Scott. Med. J. 56, 15-18 (2011).

[12] Niegsch, M., Fabritius, M.L. and Anhøj, J.: Imperfect implementation of an early warning scoring system in a Danish teaching hospital: a cross-sectional study. PloS One. 8, e70068 (2013).

[13] Navas H. and Bourdin E.: Reconstitución de un score de NEWS a partir de la creación de franjas horarias en una historia clínica electrónica. Congr. Argent. Informática En Salud 2016.

[14] The Perl Programming Language - https://www.perl.org/.

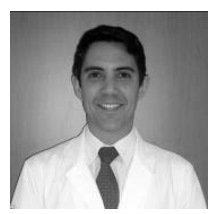

Hernán José Navas. Médico Informático de la Universidad de Buenos Aires. Profesor Adjunto Regular en la Facultad de Medicina, Universidad de Favaloro. Jefe de Informática en Salud del Sanatorio Finochietto.

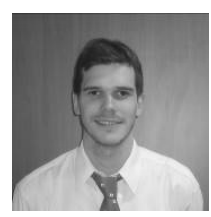

Elián Daniel Bourdin Licenciado en Bioinformática por la Facultad de Ingeniería de la Universidad Nacional de Entre Ríos (FIUNER). Es empleado del Sanatorio Finochietto en el área Informática en Salud y docente de la asignatura Informática Básica de la Universidad Favaloro. 